\title{
Sulfur Passivation of GaAsSb alloy layer Surfaces: Comparison of Ammonium Sulfide and Disulfur Dichloride Solutions
}

\author{
Shouzhu Niu \\ National Key Laboratory of High-Power \\ Semiconductor Lasers \\ Changchun University of Science and Technology \\ Changchun, China \\ nszcc@outlook.com

\section{Dan Fang*} \\ National Key Laboratory of High-Power \\ Semiconductor Lasers \\ Changchun University of Science and Technology \\ Changchun, China \\ fangdan19822011@163.com

\section{Jilong Tang} \\ National Key Laboratory of High-Power \\ Semiconductor Lasers \\ Changchun University of Science and Technology \\ Changchun, China \\ jilong_tangcust@163.com
}

\author{
Fang Fang \\ Nanchang University \\ Nanchang, China \\ fang_fang0131@126.com
}

\author{
Xuan Fang \\ National Key Laboratory of High-Power \\ Semiconductor Lasers \\ Changchun University of Science and Technology \\ Changchun, China \\ fangxuan110@hotmail.com

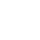

\section{Jinhua Li}

National Key Laboratory of High-Power

Semiconductor Lasers

Changchun University of Science and Technology

Changchun, China

lijinhua2000@hotmail.com

\section{Xiaohua Wang}

National Key Laboratory of High-Power Semiconductor Lasers

Changchun University of Science and Technology

Changchun, China

biewang2001@126.com

\section{Zhipeng Wei}

National Key Laboratory of High-Power Semiconductor Lasers

Changchun University of Science and Technology

Changchun, China

zhipengwei@gamil.com

\section{INTRODUCTION}

Abstract-In this paper, (NH4)2S and $\mathrm{S2Cl} 2$ are used as passivating agent for the sulphuration treatment to GaAs0.03Sb0.97. The effect of surface passivation of GaAs0.03Sb0.97 by (NH4)2S and $\mathrm{S2Cl} 2$ treatment is investigated by using photoluminescence (PL), Atomic Force Microscope (AFM). Compared with the pristine sample, the PL intensities of GaAs0.03S b0.97 that treated by (NH4)2S and $\mathrm{S2Cl} 2$ both are increased significantly. The PL intensity is strongly dependent on passivation time. For the 10s S2Cl2treated sample, the peak intensity is about 1.4 times larger than 20 min (NH4)2S-treated sample. Meanwhile, AFM images indicate that the roughness of the $\mathrm{S} 2 \mathrm{Cl} 2$ treated sample is smaller than the (NH4)2S treated sample. Thus, $\mathrm{S} 2 \mathrm{Cl} 2$ passivation is an effective method in improving the optical properties of GaAs0.03S b0.97 material.

Keywords-component; GaAsSb; Passivation; (NH4)2S; S2Cl2; Photoluminescence 
of semiconductor devices by creating Schottky metal contacts, carrier depletion, and electron-hole recombination at the surface states [7]. The high surface state densities, high surface recombination which can deeply hindered the development of GaAsSb-based devices. Therefore, it is important to control the electronic structure and surface state density to optimize the performance and the reliability of GaAsSb-based devices.

To reduce the detrimental effects of surface states on the optical and electrical properties of semiconductors, surface passivation can be applied to reduce the density of surface states. So far, many different passivation techniques have been used to overcome these surface leakages. Dielectric material deposition, sulfur passivation, over growth with wide band gap materials, and polymer film coating techniques can be listed as successful methods for passivation [8-11]. Sulfur containing solutions satisfies dangling bonds and cleans the conductive native oxides by replacing the oxygen atoms with sulfur atoms. Recent research indicates that the sulfur treatments can reduce the surface Fermi-level pinning, the surface recombination velocity (SRV) interface state density in metal-insulatorsemiconductor (MIS) and increase the intensity of photoluminescence [12-15]. Therefore, the effect of sulfur passivation of the GaAs Sb materials is deserves to further study.

In this paper, grow GaAsSb alloy layer by $\mathrm{MBE}$ and present a study of the efficacy of sulfur passivation treatment of the GaAsSb surface. The (NH4)2S and S2Cl2 solution are used to passivation and compared the results of the two methods. X-ray diffraction spectrum indicated that the crystalline quality of GaAs xSb1-x $(x=0.03)$ substrate is satisfactory. Comparing to the untreated GaAs $0.03 \mathrm{Sb} 0.97$, the PL intensity of the GaAs0.03Sb 0.97 passivated by (NH4)2S and $\mathrm{S} 2 \mathrm{Cl} 2$ both are increased significantly. However, $\mathrm{S} 2 \mathrm{Cl} 2$ treatments have a better effect than (NH4) $2 \mathrm{~S}$ treatments. Meanwhile, AFM images indicated that the roughness of the $\mathrm{S} 2 \mathrm{Cl} 2$ treated sample is better than the (NH4)2S treated sample.

\section{EXPERIMENT AL DET AILS}

The GaAsxSb1-x $(x=0.03)$ alloy described here is grown by DCA P600 solid-source molecular beam epitaxy (MBE) machine equipped with valved As and Sb crackers. The $350 \mu \mathrm{m}$ thick semi-insulating GaSb substrate to be outgassed in vacuum at $390{ }^{\circ} \mathrm{C}$ for several hours. The holder is then loaded into the growth chamber, and the oxide layer is desorbed under $\mathrm{Sb} 2$ flux by increasing the substrate temperature to $650{ }^{\circ} \mathrm{C}$. After $15 \mathrm{~min}$, the temperature dropped to $550^{\circ} \mathrm{C}$. Then, the $1 \mu \mathrm{m}$ $\mathrm{GaAsxSb} 1-\mathrm{x}$ alloy layer of is grown at $550^{\circ} \mathrm{C}$.

The as-grown $\mathrm{GaAs} x \mathrm{Sb} 1-\mathrm{x}$ is cleaved into 5 pieces and they are cleaned by acetone, ethanol and deionized water using ultrasonication for 5 minutes sequentially. Subsequently, the substrates are immersed in the $\mathrm{HCl}$ solution for $1 \mathrm{~min}$ to remove the native oxides. The samples are rinsed again in DI water and finally blown dry with compressed N2 gas. One sample is a reference sample without sulfur treatment. Two samples are soaked in $8 \%$ (NH4)2S solution for $10 \mathrm{~min}$ and $20 \mathrm{~min}$ at $60^{\circ} \mathrm{C}$. The last two samples are immersed in solution containing $\mathrm{S} 2 \mathrm{Cl} 2$ and $\mathrm{CCl} 4$ in a volume ratio $1: 3$ for $5 \mathrm{~s}$ and $10 \mathrm{~s}$ at room
temperature.Then, all the samples are rinsed using DI water and finally blown dry with compressed N2 gas.

All samples are investigated by photoluminescence spectroscopy mapping (PL, RPM 2000) and atomic force microscope (AFM,). The crystal structure of the GaAs Sb is characterized by X-ray Diffraction (Bruker AXS D8 DAVINCI) with $\mathrm{Cu}-\mathrm{Ka}$ radiation $(\lambda=1.5406 \AA)$.

\section{RESULTS AND DISCUSSION}

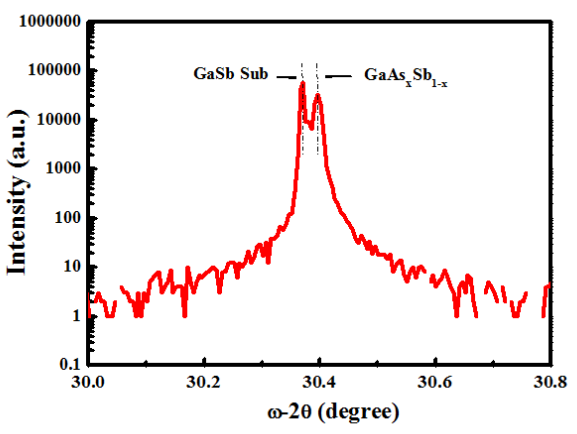

Figure 1. HRXRD pattern of GaAs0.03Sb0.97 alloy sample..

Fig. 1 shows the high resolution $\mathrm{X}$-ray diffraction (HRXRD) curve of the GaAsSb alloy samples. The diffraction peaks positioned at $30.371^{\circ}$ and $30.396^{\circ}$ is assigned to the GaSb substrate and GaAsxSb1-x alloy layer, respectively. GaAsxSb1-x alloy layer has narrower FWHM, it is indicated that the crystalline quality of the GaAs xSb1-x layer is satisfactory. The component of the GaAsxSb1-x alloy can be calculated by formula [16]:

$$
\alpha_{\text {alloy }}=\alpha_{\text {GaAs }} \mathrm{x}+\alpha_{\mathrm{GaSb}}(1-\mathrm{x})
$$

where $\alpha$ is lattice constant. The aalloy can be calculated by Bragg's law:

$$
2 \mathrm{~d} \sin \theta=\mathrm{n} \lambda
$$

where $\mathrm{n}$ is a positive integer, $\lambda$ is the wavelength of incident wave and $d$ is the interplanar distance. Due to GaAs xSb1-x alloy is cubic crystal system, the interplanar distance can be calculated by formula: $\mathrm{d}=$

Therefore, the $\mathrm{x}$ is equal to 0.03 .

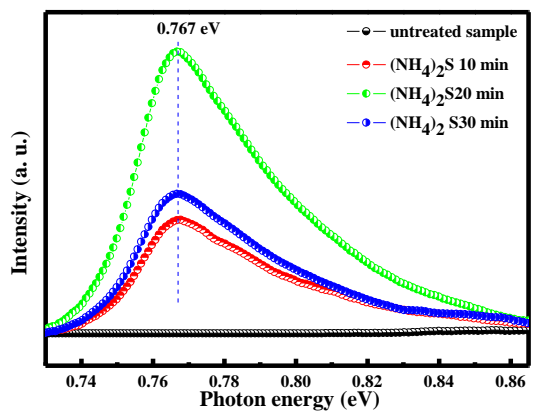




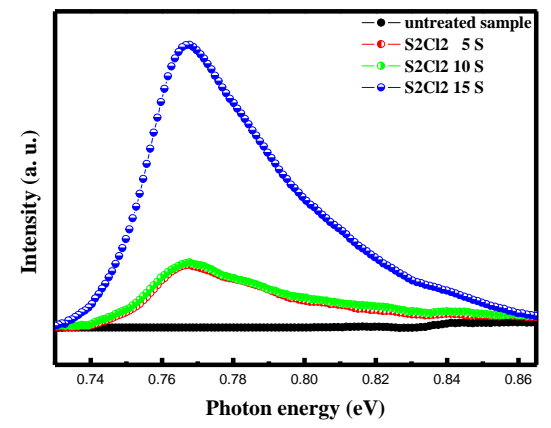

Figure 2. (a) The PL spectrum of GaAs0.03 Sb0.97 passivated by (NH4)2S for 10, 20 and $30 \mathrm{~min}$. (b) The PL spectrum of GaAs0.03Sb0.97 passivated by $\mathrm{S} 2 \mathrm{Cl} 2$ for 5,10 and $20 \mathrm{~s}$.

The energy gap of $\mathrm{GaSb}$ is $0.75 \mathrm{eV}$. By adjusting the substrate component, the band gap of GaAsxSb1-x can controlled. The band gap of GaAs xSb 1-x can be calculated by formula: $\mathrm{g} \mathrm{GaAsxSb} 1-\mathrm{x}=\mathrm{g} \mathrm{GaSb} \mathrm{x}+\mathrm{g} \mathrm{GaAs}(1-\mathrm{x})$ where $\mathrm{g}$ is band gap. The calculating data indicate that, the band gap of GaAs $0.03 \mathrm{Sb} 0.97$ is $0.767 \mathrm{eV}$. It can be observed that all PL peaks have the same position at $0.767 \mathrm{eV}$. The value of calculation is good to worth with actual measurement.

Fig. 2(a) shows the PL spectra of GaAs0.03Sb0.97 substrate and GaAs $0.03 \mathrm{Sb} 0.97$ passivated by (NH4)2S for 10,20 and $30 \mathrm{~min}$. It can be seen that the PL intensity of the GaAs0.03Sb0.97 substrate is almost zero, and (NH4)2S-treated samples showed obvious emission peaks located at $0.767 \mathrm{eV}$. This phenomenon indicates the sulfur treatment is an effective passivation method for reducing surface states density of III-V compounds. Oxide layer is very easy to form on the surface under atmospheric conditions [17]. During sulfur treatment, the sulfur atoms are strongly chemisorbed and occupied the active surface sites because of its lower heat of formation. In fact, the Obonds on the surface of the materials seem to be replaced, at least partly, by S-bonds after sulfur passivation [18].

The PL intensity is strongly dependent on (NH4) $2 \mathrm{~S}$ passivation time. Initially, the PL intensity increased with time increased. When passivation time is $20 \mathrm{~min}$, GaAs0.03Sb0.97 sample had the strongest PL emission. Then, the PL intensity decreased with the passivation extending. It is due to forming a thick sulfur ato mic layer to reduce the recombination centers of excitions $[19,20]$. As shown in the Fig. 2(b), the passivation by $\mathrm{S} 2 \mathrm{Cl} 2$ solution has the same effect. By $\mathrm{S} 2 \mathrm{Cl} 2$ solution passivation, the PL intensity of the GaAs0.03Sb0.97 surface has been enhanced obviously. When passivation time is 10 s, the PL intensity reaches a maximum and then the PL intensity dropped swiftly.
$\square\left(\mathrm{NH}_{4}\right)_{2} \mathrm{~S} \quad \square \mathrm{S}_{2} \mathrm{Cl}_{2}$

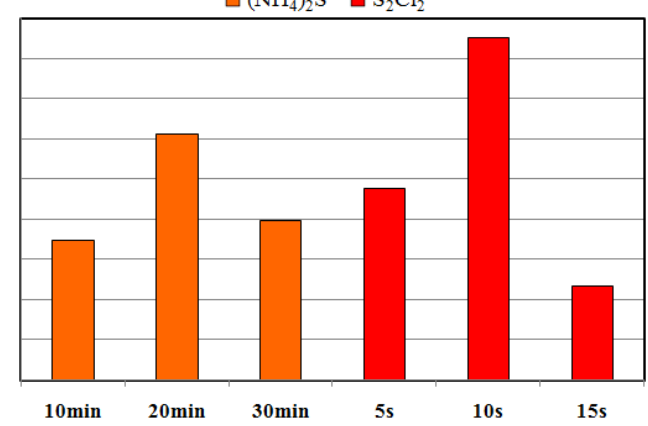

Figure 3. Relative PL peak intensity of the samples in different passivation conditions

Fig. 3, it can be seen that the PL intensity of the GaAs0.03Sb0.97 surface which are treated by (NH4)2S and $\mathrm{S} 2 \mathrm{Cl} 2$ solution. The peak intensity of the $10 \mathrm{~s} \mathrm{S2Cl2-}$ treated sample is about 1.4 times larger than the $20 \mathrm{~min}$ (NH4)2S-treated sample. Compare with (NH4)2S treatments, $\mathrm{S} 2 \mathrm{Cl} 2$ treatments have the higher PL intensity and shorter immerse time. And, $\mathrm{S} 2 \mathrm{Cl} 2$ passivation solution don't need to heat. However, $\mathrm{S} 2 \mathrm{Cl} 2$ treatments are more susceptible to the passivation time than (NH4)2S treatments. The time control of $\mathrm{S} 2 \mathrm{Cl} 2$ passivation for samples needs exceedingly accurate.
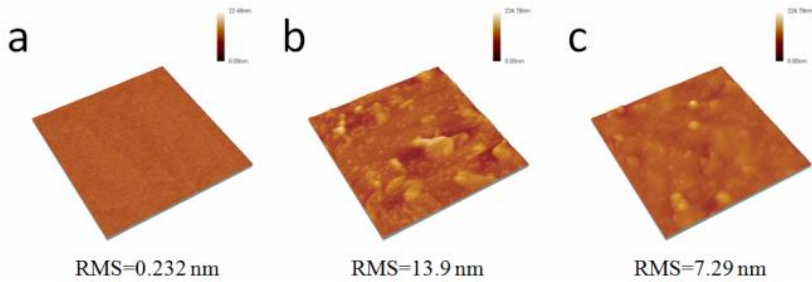

Figure 4. Measured by the AFM surface roughness of the sample icon.(a) untreated, (b)passivated by (NH4)2S for $20 \mathrm{~min}$, (c)passivated by $\mathrm{S} 2 \mathrm{Cl} 2$ for $10 \mathrm{~s}$.

Choose the samples with strongest PL intensity in two methods to be compared. Fig. 4 shows the AFM test results of GaAs0.03 Sb0.97 substrate, (NH4)2S-treated and $\mathrm{S} 2 \mathrm{Cl} 2$-treated samples. It is clearly that the surface of GaAs0.03Sb0.97 substrate is very smooth. The surface roughness of RMS (Root Mean Square) is $0.232 \mathrm{~nm}$. The surface morphologies of passivated GaAs0.03Sb0.97 sample became rougher. For the $20 \mathrm{~min}$ (NH4)2S-treated sample and the $10 \mathrm{~s} \mathrm{S2Cl2-treated} \mathrm{sample,} \mathrm{the} \mathrm{RMS} \mathrm{are}$ $7.29 \mathrm{~nm}$ and $13.9 \mathrm{~nm}$, respectively. The reason of this phenomenon could be due to the etching reaction of the alkaline solution and the deposition of polycrystalline sulfur. It is clearly that the roughness of the $\mathrm{S} 2 \mathrm{Cl} 2$ treated sample is smaller than the (NH4)2S treated.

\section{CONCLUSIONS}

In order to study the properties of $\mathrm{GaAsSb}$ alloy by (NH4)2S and S2Cl2 treatment, grows GaAs0.03Sb0.97 by $\mathrm{MBE}$, then (NH4)2S and $\mathrm{S} 2 \mathrm{Cl} 2$ solution is used to passivate the surfaces of GaAsSb alloy. XRD spectrum indicated that the crystalline quality of GaAs $0.03 \mathrm{Sb} 0.97$ substrate is satisfactory. Compared with the pristine sample, the PL intensity of GaAs0.03Sb0.97 by two 
passivating agents has increased significantly. The PL intensity is strongly dependent on passivation time. For the (NH4)2S and S2Cl2 treatments, the optimum passivation time is around $20 \mathrm{~min}$ and $10 \mathrm{~s}$, respectively. The PL intensity of the $10 \mathrm{~s} \mathrm{S2Cl2-treated} \mathrm{sample} \mathrm{is} \mathrm{about} 1.4$ times larger than the $20 \mathrm{~min}$ (NH4)2S-treated sample. Meanwhile, AFM images indicated that the roughness of the S2Cl2-treated sample is smoother than the (NH4)2Streated sample. Comparing to PL intensity and surface roughness, $\mathrm{S} 2 \mathrm{Cl} 2$ treatments have a greater efficiency. However, due to more susceptible to the passivation time, $\mathrm{S} 2 \mathrm{Cl} 2$ treatment needs to be extra accurate. In conclusion, $\mathrm{S} 2 \mathrm{Cl} 2$ passivation is a more effective method in improving the optical properties of GaAs0.03Sb0.97 material, which is then conducive to the development of $\mathrm{GaAsSb}$-based optoelectronic devices.

\section{ACKNOWLEDGMENT}

This work is supported by the National Natural Science Foundation of China (61076039, 61204065, 61205193, 61 307045, 61404009, 61474010), Research Fund for the Doc toral Program of Higher Education of China (20112216120 005), the Developing Project of Science and Technology o f Jilin Province (20140520107JH, 20140204025GX), Nati onal Key Laboratory of High Power Se miconductor Lasers Foundation(No.9140C310101120C031115,9140C310104 110C3101,9140C310102130C31107, 9140C31010240C310004).

\section{REFERENCES}

[1] Lebedev, M. V., Kunitsyna, E. V., Calvet, W., Mayer, T., \& Jaegermann, W, "Sulfur passivation of $\mathrm{GaSb}$ (100) surfaces: comparison of aqueous and alcoholic sulfide solutions using synchrotron radiation photoemission spectroscopy," The Journal of Physical Chemistry C, 117(31), July 2013, pp. 15996-16004. doi: $10.1021 / \mathrm{jp} 401942$ p.

[2] Canedy, C., Boishin, G., Bewley, W., Kim, C., Vurgaftman, I., Kim, M., Whitman, L. "Correlating growth conditions with photoluminescence and lasing properties of mid-IR antimonide type II "W" structures," Journal of Vacuum Science \& Technology B, 22(3), June 2004, pp. 1575-1579, doi: 10.1116/1.1688805.

[3] Steinle, G., Riechert, H., \& Egorov, A. Y., "Monolithic VCSEL with InGaAsN active region emitting at $1.28 \mu \mathrm{m}$ and $\mathrm{CW}$ output power exceeding $500 \mu \mathrm{W}$ at room temperature," Electronics Letters, 37 (2), January 2001, pp.93-95, doi: 10.1049/el:20010098.

[4] Yamada, M., Anan, T., Kurihara, K., Nishi, K., Tokutome, K. Kamei, A., \& Sugou, S., "Room temperature low-threshold CW operation of $1.23 \mu \mathrm{m}$ GaAsSb VCSELs on GaAs substrates," Electronics Letters, 36(7), March 2000, pp. 637-638, doi: 10.1049/el:20000483.

[5] Liu, Z., Hawkins, B., \& Kuech, T, Chemical and structural characterization of $\mathrm{GaSb}$ (100) surfaces treated by $\mathrm{HCl}$-based solutions and annealed in vacuum. Journal of Vacuum Science \& Technology B, 21(1), December 2003, pp. 71-77, doi: 10.1116/1.1532023.

[6] Lin, C. L., Su, Y. K., Se, T. S., \& Li, W. L, "Variety transformation of compound at GaSb surface under sulfur passivation," Japanese Journal of Applied Physics, 37(12B), December 1998, pp.L1543-L1545, doi: 10.1143/JJAP.37.L1543
[7] Tajik, N., Haapamaki, C., \& LaPierre, R., "Photoluminescence model of sulfur passivated p-InP nanowires," Nanotechnology, 23(31), August 2012, pp.315703-315708, doi:10.1088/09574484/23/31/315703

[8] Gin, A., Wei, Y., Bae, J., Hood, A., Nah, J., \& Razeghi, M. , "Passivation of type II InAs/GaSb superlattice photodiodes," Thin Solid Films, Volumes 447-448, January 2004, pp. 489-492, doi: 10.1016/j.tsf.2003.09.00

[9] Plis, E., Rodriguez, J.-B., Lee, S., \& Krishna, S. (2006). Electrochemical sulphur passivation of $\mathrm{InAs} / \mathrm{GaSb}$ strain layer superlattice detectors. Electronics Letters, 42(21), October 2006, pp. 1248-1249, doi: 10.1049/el:20062495.

[10] Rehm, R., Walther, M., Fuchs, F., Schmitz, J., \& Fleissner, J. (2005). Passivation of InAs/(GaIn) Sb short-period superlattice photodiodes with $10 \mu \mathrm{m}$ cutoff wavelength by epitaxial overgrowth with AlxGa1-xAsySb1-y. Applied Physics Letters, 86(17), April 2005, pp. 173501-173503, doi: 10.1063/1.1906326.

[11] Hood, A., Delaunay, P.-Y., Hoffman, D., Nguyen, B.-M., Wei, Y., Razeghi, M., \& Nathan, V., "Near bulk-limited R0A of longwavelength infrared type-II InAs/GaSb superlattice photodiodes with polyimide surface passivation," Applied Physics Letters, 90(23),June 2007, pp. 233513.1-233513.3, doi: 10.1063/1 .2747172.

[12] Martens, K., Wang, W., De Keersmaecker, K., Borghs, G., Groeseneken, G., \& Maes, H., "Impact of weak Fermi-level pinning on the correct interpretation of III-V MOS CV and GV characteristics," Microelectronic engineering, 84(9), October 2007, pp. 2146-2149, doi:10.1016/j.mee.2007.04.039

[13] Zhao, H., Shahrjerdi, D., Zhu, F., Kim, H.-S., Injo, O., Zhang, M., Lee, J. C., "Inversion-type InP MOSFET s with EOT of $21 \AA$ using atomic layer deposited $\mathrm{A} 2 \mathrm{O} 3$ gate dielectric," Electrochemical and solid-state letters, 11(8), June 2008, H233-H235, doi:10.1149/1.2938728.

[14] Yablonovitch, E., Sandroff, C., Bhat, R, \& Gmitter, T., "Nearly ideal electronic properties of sulfide coated GaAs surfaces," Applied Physics Letters, 51(6), June 1987, pp. 439-441, doi: 10.1063/1.98415.

[15] Buehlmann, P., Bailat, J., Dominé, D., Billet, A., Meillaud, F., Feltrin, A., \& Ballif, C, "In situ silicon oxide based intermediate reflect or for thin-film silicon micromorph solar cells," Applied Physics Letters, 91(14), October 2007, pp. 143505.1-143505.3, doi: 10.1063/1.2794423.

[16] Villaggi, E., Bocchi, C., Armani, N., Carta, G., Rossetto, G., \& Ferrari, C, "Deviation from vegard law in lattice-matched InGaAs/InP epitaxial structures," Japanese Journal of Applied Physics, 41(2S), February 2002, pp. 1000-1003, doi:10.1143/JJAP.41.1000.

[17] Salesse, A., Alabedra, R., Chen, Y., Lakrimi, M., Nicholas, R, Mason, N., \& Walker, P., "Improved photoluminescence from electrochemically passivated GaSb," Semiconductor science and technology, 12(4), April 1997, pp. 413-418, doi:10.1088/0268$1242 / 12 / 4 / 013$.

[18] Dutta, P., Sangunni, K., Bhat, H., \& Kumar, V., "Sulphur passivation of gallium antimonide surfaces," Applied Physics Letters, 65(13), July 1994,pp. 1695-1697, doi: 10.1063/1.112889.

[19] Eftekhari, G., "Electrical properties of sulfur-passivated III-V compound devices," Vacuum, 67(1), September 2002, pp. 81-90, doi:10.1016/S0042-207X(02)00195-1.

[20] Chioncel, M., Díaz-Guerra, C., \& Piqueras, J., "Luminescence from indented Te-doped GaSb crystals," Semiconductor science and technology, 19(3), March 2004 , pp. 490-493, doi:10.1088/0268-1242/19/3/036. 